\title{
Study on the Efficacy and Safety of Total Laparoscopic Radical Resection and Open Surgery for Rectal Cancer
}

\author{
$\mathrm{Yi} \mathrm{He}^{1}$, Linsen Shi ${ }^{2}$, Dongsheng $\mathrm{He}^{2}$ \\ ${ }^{1}$ Institute of Clinical, Xuzhou Medical University, Xuzhou, Jiangsu, 221004 \\ ${ }^{2}$ Department of Gastrointestinal Surgery, Affiliated Hospital of Xuzhou Medical University, Xuzhou, Jiangsu, \\ 221006
}

Keywords: Total Laparoscopic Radical Resection, Open Surgery, Rectal Cancer

\begin{abstract}
Objective: To study the efficacy and safety of total laparoscopic radical resection of rectal cancer and open surgery for rectal cancer. Methods: 120 patients with rectal cancer who were treated in our hospital from January 2013 to December 2016 were divided into two groups according to the surgical procedure. 60 patients were treated with open rectal cancer radical surgery (control group), and 60 patients were given full Laparoscopic radical resection of rectal cancer (observation group), comparing the efficacy and safety of the two groups. Results: The operation time of the observation group was significantly higher than that of the control group $(\mathrm{P}<0.05)$. The recovery time of bowel movement, anal exhaust time, feeding fluid time, indwelling catheter time and postoperative hospital stay were significantly shorter than the control group. <0.05). The intraoperative blood loss, incision pain index and total length of the incision in the observation group were significantly lower than those in the control group $(\mathrm{P}<0.05)$. The incidence of wound infection and intestinal obstruction in the observation group was significantly lower than that in the control group $(\mathrm{P}<0.05)$. The incidence of postoperative complications in the observation group was significantly lower than that in the control group $(\mathrm{P}<0.05)$. The recurrence rate of patients in the observation group was slightly lower than that of the control group $(\mathrm{P}>0.05)$. The 1-year and 3-year survival rates of the observation group were slightly higher than those of the control group $(\mathrm{P}>0.05)$.
\end{abstract}

\section{Introduction}

Rectal cancer belongs to a class of malignant tumors. Its pathogenesis is the continuous proliferation of malignant rectal cells in the body. In recent years, the incidence of rectal cancer has reached the third place in cancer incidence, affecting the safety of patients, and targeting the malignant tumor. Effective treatment methods are also being studied. Patients do not show obvious symptoms in the early stages of rectal cancer. Therefore, they are usually in the middle or late stage when the disease is diagnosed. Therefore, it is necessary to take correct and effective treatment. Safeguard the lives of patients. With the development of medical technology, the current treatments for rectal cancer mainly include traditional open radical resection of rectal cancer and total laparoscopic radical resection of rectal cancer. The former causes great trauma to the patient during the operation. And prone to complications, the latter belongs to a new type of surgical treatment, the treatment effect is more obvious.

\section{Data and methods}

In July 2014, July 2016, 54 patients who underwent open surgery in our hospital were treated with open surgery for group A. In the same period, 54 patients with rectal cancer were selected for total laparoscopic radical resection. For group B. Group A: 25 males and 29 females, aged from 32 to 70 years old, with an average of $(50.4 \pm 4.5)$ years; Group B males and 24 females, aged 35 to 69 years, average It is $(49.8 \pm 4.9)$ years old. There was no significant difference between the two groups in general data $\mathrm{P}>0.05$. Before the operation, the two groups of patients were fasted for six hours, preoperative preparation, tracheal intubation and general anesthesia. Group B underwent 
total laparoscopic radical resection of rectal cancer. The main step was to keep the patient's position in the low-foot position, select the lithotomy position, and perform a longitudinal arc-shaped incision on the upper edge of the umbilical ring to establish the artificial pneumoperitoneum. Laparoscopy was performed to observe the location of the tumor and the surrounding conditions. The incision was placed into the operation instrument, the left colonic peritoneum was cut with an ultrasonic knife, and the laparoscope was used to guide, and the layers of the mesorectum were slowly separated layer by layer, and the rectum was completely free. The distal rectal margin was performed according to the distance between the tumor and the anus. Clean the distal intestine and select sterile distilled water. Use a stapler to break the intestinal lumen, enlarge the puncture hole, insert the protective sleeve, remove the tumor through the protective sleeve, remove the lymph nodes around the tumor, and then insert the stapler from the puncture hole. After tightening, rebuilding the pneumoperitoneum, and completing the anastomosis of the colon and anal canal, place a drainage tube at the anastomosis incision to complete the operation. In group A, conventional open rectal cancer was performed. The incision was selected as the lateral rectus abdominis and the middle of the abdomen. The other procedures were the same as those in group B.

Observation indicators (1) Statistics and comparison between the two groups of operation time, intraoperative blood loss, intestinal peristalsis recovery time, anal exhaust time, feeding fluid time, indwelling catheter time, postoperative hospital stay, incision pain index, total length of incision. (2) The main complications of the two groups (incision infection, intestinal obstruction, anastomotic bleeding, pulmonary infection, puncture fistula, anastomotic leakage, abdominal hemorrhage and subcutaneous emphysema) were observed. The incidence of complications was compared between the two groups. (3) Record the recurrence and survival of the two groups at the first and third years of follow-up, and compare the recurrence rate and survival rate between the two groups.

The data were statistically analyzed using SPSS 19.0 software. The measurement data were expressed by (x- $\pm \mathrm{s})$ and compared by t test; the count data was expressed by rate (\%), and the word 2 test was used for comparison. The difference was statistically significant at $\mathrm{P}<0.05$.

\section{Results}

The operation time of the observation group was significantly higher than that of the control group $(\mathrm{P}<0.05)$. The recovery time of bowel movement, anal exhaust time, feeding fluid time, indwelling catheter time, and postoperative hospital stay were significant in the observation group. Shorter than the control group $(\mathrm{P}<0.05)$. The intraoperative blood loss, incision pain index and total length of the incision in the observation group were significantly lower than those in the control group $(\mathrm{P}<0.05)$. The incidence of postoperative complications in the two groups was significantly lower than that in the control group, but the difference was not statistically significant $(\mathrm{P})$. $>0.05)$. The total postoperative complication rate was $8.33 \%$ in the observation group, which was significantly lower than that in the control group (28.33\%). The difference was statistically significant $(\mathrm{P}<0.05)$. The incidence of incision infection in the observation group was 0 , which was significantly lower than $6.67 \%$ in the control group. The incidence of intestinal obstruction in the observation group was 0 , which was significantly lower than that in the control group (8.33\%). The difference between the two groups was statistically significant $(\mathrm{P}<0.05)$. .

\section{Discussion}

Rectal cancer has a very high mortality rate, and advanced rectal cancer may cause the metastasis of organ cancer cells, which may seriously affect the function of the body. At present, people's living habits and eating habits are mostly unhealthy, and a large amount of protein and fat are ingested, and the intake of crude fiber components is gradually reduced, leading to rectal cancer. In the clinic, the main way to treat rectal cancer is surgery. With the development of medical technology, laparoscopic surgery for rectal cancer has been widely used in clinical practice. Laparoscopic radical resection of rectal cancer before lymph node metastasis can reduce mortality and improve long-term survival. The end of the laparoscopic structure is a lens. When applied 
during surgery, it has a clearer view of the surgery. It can observe the internal situation from all angles, and it is conducive to precise operation through clear vision. With the development of minimally invasive techniques, surgical procedures have gradually developed into minimally invasive treatments. Advanced medical devices such as staplers and ultrasonic scalpels have been gradually applied in the treatment of rectal cancer. In minimally invasive surgery, the trauma to patients is small, and the intraoperative field of vision is small. Clear, more precise operation, avoiding damage to the nerves and blood vessels of surrounding tissues, and help prevent postoperative complications.

Studies have shown that laparoscopic radical resection of rectal cancer has a great advantage in the treatment of rectal cancer patients. Under the guidance of laparoscopy, the trauma is small, the interference to the basin and abdominal organs is small during the operation, and the ultrasonic scalpel is applied during the operation. The intraoperative blood loss is small, the degree of postoperative pain and recovery rate is much higher than that of open surgery. The laparoscopic field of vision is clearer and wider, and small lesions that are not observed during open surgery can be found, so that the lesion can be more thoroughly removed. It is conducive to improving the surgical results. At present, laparoscopic radical resection of rectal cancer has gradually become the preferred method for the treatment of rectal cancer patients. Laparoscopic radical resection of rectal cancer has the following advantages compared with open surgery: (1) intraoperative trauma is small, it will not directly expose important organs and reduce postoperative infection; (2) laparoscopic guidance, the surgical field of vision is clearer and broader Therefore, the operation is more precise and the interference to the organs is small; (3) The intraoperative ultrasound knife is used to reduce the amount of intraoperative blood loss, and the postoperative patient has less pain, which is beneficial for postoperative recovery.

In this study, the operation time of the observation group was significantly higher than that of the control group $(\mathrm{P}<0.05)$. The recovery time of bowel movement, anal exhaust time, fluid intake time, indwelling catheter time, and postoperative hospital stay were significantly shorter in the observation group. Control group $(\mathrm{P}<0.05)$. The intraoperative blood loss, incision pain index and total length of the incision in the observation group were significantly lower than those in the control group $(\mathrm{P}<0.05)$. Probably because the surgical field of laparoscopic surgery provides clearer vision, and the specific tissues and organs in the surgical field can be clearly displayed on the screen, which is conducive to the accurate separation of blood vessels and nerves. At the same time, ultrasonic scalpel is used in the operation to make hemostasis more Thoroughly, the vascular cutting closure makes the blood vessel ligation accurate, which can effectively prevent blood vessel and wound bleeding, and reduce the amount of intraoperative blood loss. Laparoscopic radical resection of rectal cancer has less trauma to the patient, and the postoperative patient has less pain, which is conducive to postoperative recovery, shortening the time of patient getting out of bed and recovery time of peristalsis. Laparoscopic radical resection of rectal cancer is complicated and requires careful operation, so the operation time is relatively long.

The radical nature of the tumor requires the complete removal of regional lymph nodes. The third lymph node of rectal cancer is the main lymph node. In the surgical treatment, the main lymph nodes need to be completely removed. In this study, there was no significant difference in the number of lymph nodes in the two groups $(\mathrm{P}>0.05)$. The results show that laparoscopic radical rectal cancer surgery, like traditional open surgery, can accurately remove the primary tumor anatomically, and also accurately remove regional lymph nodes. In this study, the number of lymph nodes in the observation group was slightly higher than that in the control group $(\mathrm{P}>0.05)$. The results showed that laparoscopic radical resection of rectal cancer could completely and completely remove the lymph nodes in the rectum. In this study, the incidence of anastomotic bleeding, pulmonary infection, puncture, anastomotic leakage, abdominal hemorrhage, and subcutaneous emphysema was lower in the observation group than in the control group, but the difference was not statistically significant $(\mathrm{P}>0.05)$. The total postoperative complication rate was $8.33 \%$ in the observation group, which was significantly lower than that in the control group (28.33\%). The difference was statistically significant $(\mathrm{P}<0.05)$. The incidence of incision infection in the 
observation group was 0 , which was significantly lower than $6.67 \%$ in the control group. The incidence of intestinal obstruction in the observation group was 0 , which was significantly lower than that in the control group (8.33\%), and the difference was statistically significant $(\mathrm{P}<0.05)$. During open surgery, the intestinal tract is affected to activate neutrophils and macrophages, resulting in the release of a large number of inflammatory mediators. Intestinal peristalsis and contraction of smooth muscle were inhibited, resulting in intestinal obstruction in a small number of patients. Laparoscopic radical resection of rectal cancer is ongoing, the interference to the intestinal tract is lighter, the intestinal inflammatory response is reduced, and the patient can get out of bed early after surgery, and accelerate the recovery of gastrointestinal function as soon as possible, so intestinal obstruction The incidence is low. Laparoscopic radical resection of rectal cancer has less trauma and quick healing after surgery, so the incidence of wound infection is lower than that of open surgery.

\section{Conclusion}

The development of medical technology has promoted the application of total laparoscopic radical resection of rectal cancer. It is mainly to perform radical laparoscopic radical resection before the lymph node metastasis of rectal cancer patients. Laparoscopy can expand the surgical field during surgery. The internal situation can be observed in all aspects and at multiple angles, which is conducive to the operation, avoid unnecessary trauma to the patient during the operation, and is beneficial to the patient's later recovery and reduce the cancer mortality. This study used conventional radical rectal cancer radical resection and total laparoscopic rectal cancer radical resection for rectal cancer patients. The results showed that total laparoscopic radical resection of rectal cancer is more dominant, with fewer complications, in laparoscopic surgery. The operation under the operation has less interference to the organs such as the basin and the abdominal cavity. At the same time, the ultrasonic scalpel used for surgery can reduce the trauma to the patient and reduce the postoperative complications of the patient, so that it can be treated in patients with rectal cancer. Preference is given to total laparoscopic radical resection of rectal cancer to improve treatment rate.

\section{Acknowledgements}

Fund Project: Subject of Xuzhou Science and Technology Bureau Project No.: KC14SH118

\section{References}

[1] Xiao Yi, Liu Lin, Liu Peihua, et al. Clinical efficacy of laparoscopic radical resection for rectal cancer and radical resection of rectal cancer[J]. Journal of Qiqihar Medical College, 2015, 36(17): 2582-2583.

[2] Zhou Jing. Surgical cooperation of laparoscopic radical resection for rectal cancer [J]. Psychologist, 2015, 21 (6): 57-58.

[3] Fang Zhuangwei, Yuan Bo, Zhou Weiping, et al. Safety and long-term efficacy of open surgery and laparoscopic surgery for rectal cancer [J]. Chinese Journal of Clinical Research, 2016, 29(10): 1375-1377.

[4] Wang Xiongfei, Liu Chunqing, Feng Yanyu, et al. Comparison of laparoscopic rectal cancer surgery and traditional open surgery for rectal cancer [J]. People's Military Medical University, 2014, (10): 1070-1071.

[5] Huang Yan. Clinical analysis of laparoscopic and open surgery for rectal cancer [J]. Modern Journal of Integrated Traditional Chinese and Western Medicine, 2013, 22 (30): 3368-3370. 